Hence we get for the phase $\delta_{3}$ this expression-

$$
\tan \delta_{3}=-\frac{\frac{\cos m r}{m r}-\frac{\sin m r}{m^{2} r^{2}}}{\frac{\sin m r}{m r}+\frac{\cos m r}{m^{2} r^{2}}}
$$

or, after transformation-

$$
\delta_{3}=m r-\tan ^{-1} m r .
$$

The line $\delta_{3}$ of our Fig. 5 represents this function. One sees that for this action the phase steadily increases direct from the origin. Those phenomena, therefore, which indicate a finite pace of propagation must make themselves apparent by interferences even close to the vibrator. So it shows itself in these experiments, and just herein consists the advantage which we derived from this kind of interference experiment. But the apparent velocity comes out greater in the neighbourhood of the vibrator than at a distance, and it is not to be denied that the phase of the interference must theoretically change less, but notably more quickly than was experimentally the case.

It appears to me probable that a more complete theory, one which does not consider both conductors as vanishingly small -perhaps, also, another estimate of the value of $\lambda$-would here afford a better correspondence.

It is of importance that even on Maxwell's theory the experiments cannot be explained without assuming a marked difference between the velocity of waves along wire and their velocity in free space.

(To be continued.)

\section{NOTE ON THE USE OF GEISSLER'S TUBES FOR DETECTING ELECTRICAL OSCILLA- TIONS.}

$\mathrm{A}^{\mathrm{T}}$ the suggestion of Prof. Lodge, I undertook to repeat in the Physical Laboratory of the University College, Liverpool, Hertz's celebrated experiments on electrical oscillations.

In performing these experiments, I was searching for means to make the effect of the electrical oscillations more easily observable, and I was induced to use for this purpose (I) Geissler's tubes, in order to strengthen the visible effect; and (2) the chemical action of the oscillating currents (paper soaked in solution of iodide of potash), in order to obtain a permanent trace of them.

For the present I will describe briefly the results of the use of Geissler's tubes.

In order to produce the electrical oscillations, I used a conductor consisting of two zinc plates, about $4 \mathrm{I} \cdot 5$ centimetres square, suspended in the same plane 55 centimetres apart; to each plate was fastened a No. 6 copper wire, which was finisher off with a small brass knob. The two brass knobs were about 5 millimetres apart, and formed the sparking gap, as we shall call it. As receiver of the oscillations, I used, like Hertz, circles of No. 14 wire, 35 centim etres in radius.

After the example of Mr. F. T. Trouton (NATURE, February 21, p. 39r), I will call the first conductor a vibrator, and the wire circles, or other receivers, resonators.

The vibrator was connected with a small coil, 20 centimetres long, supplied with an ordinary spring interrupter, and excited by four secondary cells.

If we connect one electrode of a convenient Geissler's tube with either side of the sparking gap of the resonator, currents pass through or into the tube, which lights up and so makes the effect of the electrical oscillations on the resonator visible even at a great distance.

Of the few tubes which were at my disposal, I found that the most convenient for this purpose was a small one with electrodes 8.5 centimetres apart, and filled with highly rarefied air. But spectral tubes 20 centimetres long and filled with bydrogen, oxygen, or nitrogen also gave good results.

With the first mentioned tube I perceived a visible effect, when the resonator was held horizontally in the plane containing the wires of the vibrator, and with the sparking gap turned towards it, at a distance of 4 metres from the vibrator. By this arrangement all the phenomena described by Hertz (Wiedemann's Ann., xxxiv. p. 160,1888 ) about the direction of the electrical lines of force can easily be shown.

A very instructive experiment is to show the directions of these lines by several resonators disposed round the vibrator. For this purpose I suggest the following apparatus :-
On a wooden frame mounted so as to be able to revolve on a vertical axis standing under the sparking gap of the vibrator are fastened several resonators, with their planes vertical and parallel respectively to the directions of the lines of force and the sparking gaps at the highest point. These resonators are supplied with Geissler's tubes. In this position of the resonators all the tubes will lighten up when the vibrator is working. But if the frame with the resonators moves round the vertical axis, the light of the tubes will become weaker, and, when the frame is turned $90^{\circ}$, the tubes will become quite dark; the planes of all the vibrators in this position being perpendicular to the directions of the lines of force. This change will occur inversely by turning the frame from $90^{\circ}$ to $180^{\circ}$

If, instead of one resonator, two are fastened to each point of the frame, one perpendicularly to the other, both being vertical, the changes in either of these will be contrary-that is to say, when the light in one set of the tubes becomes brighter it becomes weaker in the set perpendicular to it and vice versâ. Thus the strength of the light is, so to say, proportional to the magnitude of the components of the lines of force in the direction of the tubes.

If a disconnected Geissler's tube is held near the vibrator, it begins in a short time to light up, owing to oscillatory currents passing through it. The same effect is obtained if instead of holding the tube by the hand it reposes on an insulating body. This lighting occurs at all points near the vibrator, except about the sparking gap. The tube becomes quite dark if the hand or a conductor is interposed between it and the vibrator; on the contrary, the interposing of an insulating body causes no change in the tube. The tube becomes more sensitive if a portion of it is surrounded with tinfoil.

In this way the existence of electrical oscillations in space can be ascertained, and also the transparency of insulating bodies and the opacity of conductors for electrical oscillations can be demonstrated.

When the two electrodes of a Geissler's tube are connected with two different points of a resonator, the effect in the tube is produced by the difference of potential of the two points. If now we connect one point of the vibrator or the resonator with one electrode of the tube, the other electrode hanging free in the air or being earthed, we have an alternative current through the tube whenever the potential of the point connected with the electrode becomes different from zero, and thus the tube lights up. The effect is strengthened if one portion of the tube is surrounded by tinfoil. This is a very convenient arrangement for observing the form of the electrical oscillation in conductors.

If we investigate in this manner our circular resonator held vertically before the vibrator, with its plane parallel to it and the sparking gap upwards, we find that a tube banging at the lower end of the vertical diameter of the circle, opposite to the sparking gap, remains quite dark, and lights up when moved to the right or to the left of this point. The light becomes brighter till the horizontal diameter is reached; further on the light begins to grow weaker till the sparking gap is attained, where the tube, however, continues to lighten. The light becomes weaker when the sparking gap is narrowed, and ceases when it is quite closed. Thus we see that the circular resonator possesses one node at its lowest point, two ventral segments at equal distances from the node and the sparking gap, and two minima of oscillation one on each side of the sparking gap.

That a node is situated at the point opposite to the sparking gap is also ascertained by observing that by touching this point with the finger or by hanging from it a piece of wire or by connecting it to earth, no change is to be remarked in the spark of the resonator. These manipulations, if applied to another point of the resonator, diminish the spark.

If the resonator is formed by a closed circle of wire, we find a node at each end of the vertical, and a segment at each end of the horizontal, diameter of the circle. The distance between the two nodes being here IIO centimetres, the wave-length is 220 centimetres, while the length of the primary wave is about 880 centimetres. Thus the wave-length in the resonator corresponds to the second higher octave of the fundamental oscillation.

If, instead of circular, we use linear resonators placed parallel to the vibrator, we must be very careful to distinguish between the effect produced directly from the vibrator in the Geissler tube and the effect caused by the oscillations of the resonator. In the case of the circular resonators, placed in the position above described, one need not trouble much about the direct effect of the vibrator, this being very small in the neighbourhood of the vibrator's sparking gap. 
To prevent the direct action a small uninsulated metal screen can be placed between the vibrator and the tube, or the tube can be hung by a long and fine wire, in order to be removed from the sphere of the direct action of the vibrator. The best plan is, however, to surround the tube by wire gauze, which stops the direct action of the vibrator on the tube, and yet permits the tube to be observed. The absence of direct action can be ascertained in the different positions which the tube takes by insulating it from the resonator without changing its position, and noticing if it becomes quite dark.

If we place before the vibrator a resonator, consisting of a straight wire 220 centimetres long (I used copper wire No. 6), we find, by the tubes, that nodes exist in the middle and the two ends of the wire, consequently two segments at 55 centimetres from each end of the wire.

(The above-described circular resonator can be likened to a linear one which is curved to a circle and its two ends soldered together, thus the two nodes of the ends becoming one single node.)

If this straight wire is cut in the middle, a torrent of sparks passes between the separated ends, even if they are removed several millimetres apart. If then we examine each half of the wire, we find that it possesses a node in the middle and two segments, each at one end, but the node is not so well defined as in the case of the uncut wire; there is no single point the potential of which remains continually equal to zero, but a line in which the difference of potential from zero is a minimum. This complicated form of oscillation is produced by the fact that the forces acting in this resonator are not equal at all points or symmetrically distributed with respect to it, as in the case of the long resonator. The oscillations of the short resonator may be compared to those of a rod which is not firmly fixed by its middle. The state of these oscillations is not stable. If one or both ends of this resonator are touched by the finger, they become nodes, and a well-defined segment appears at the middle of the resonator. If the Geissler tube be connected with this middle point, it begins to light up when the ends of the resonator are touched, and ceases to light the moment the fingers are removed; the contrary takes place if the tube be connected with one end of the resonator. This phenomenon is analogous to the change of the form of the vibration of a rod when fixed by its middle or by its ends.

Quite similar is the mode of oscillation of a resonator 220 centimetres long disposed on one side of the vibrator; it possesses also a node at the middle not well defined, and a segment at each end.

To conclude, I will describe the mode of oscillation of a resonator, I Io centimetres long, disposed parallel and symmetrically to the vibrator. This resonator possesses one node in the middle, very clearly defined, and a segment at eitler end. This form of oscillation is the same as would occur in the long resonator if one-fourth of its length from each end were cut off.

In the case of the latter resonator and of the long one, which is also symmetrically disposed to the vibrator, the oscillations are very stable, and much stronger than in the case of the resonators placed on one side of the vibrator. The mode of the oscillations of these symmetrical resonators is not disturbed by touching them by the fingers at any point, although the mode of oscillation is disturbed if we touch the unsymmetrical resonators at any point whatever.

The experiments described must be performed in a dark room, and much care be used in the choice of the proper Geissler tubes. Tubes containing mercury are very sensitive, and they become more so if the mercury be allowed to flow several times from one end of the tube to the other.

Liverpool, March.

E. T. Dragoumis.

\section{SOCIETIES AND ACADEMIES. LONDON.}

Royal Society, March 21.-- "An Experimental Investigation of the Circumstances under which a Change of the Velocity in the Propagation of the Ignition of an Explosive Gaseous Mixture takes place in Closed and Open Vessels. Part I. Chronographic Measurements." By Frederick J. Smith, M.A., Millard Lecturer on Mechanics, Trinity College, Oxford. Communicated by A. (r. Vernon Harcourt, F.R.S.

It has been noticed by several investigators, vic. MM. Berthelot and Vielle, MM. Mallard and Le Chatelier, and Prof. H. B. Dixon, F.R.S., that explosive gaseous mixtures after ignition do not reach their maximum velocity of propagation at once, but that a certain maximum velocity is attained soon after initial ignition.

In order to investigate this period, which may be called the acceleration period of an explosion, chronographic measurements of a peculiar nature were found necessary.

It was at once evident that but little advance in this branch of the subject of explosions could be made unless exceedingly minute periods of time could be measured with certainty.

A new form of chronograph has been devised to meet as far as possible all the requirements of the case, by means of the instrument. The following results have been obtained :-

(I) The $\frac{1}{200 \sigma}$ of a second can be measured with ease, and periods of time differing from $\frac{1}{10}$ of a second to $\frac{1}{20000}$ of a second can be recorded on the same moving surface.

(2) The surface which receives the record moves at a velocity which is practically constant during the traverse of $5 \mathrm{~cm}$.; also its velocity can be varied between wide limits.

(3) A large number of time records can be made side by side, all records being made in straight lines.

(4) Fractions of recorded vibrations of a fork can be subdivided by means of a micrometer microscope. This is not the case with vibrations recorded on a surface attached to a pendulum, where the velocity varies from zero up to a maximum at the middle of the swing.

The electro-magnetic styli, by means of which events are marked, are so constructed that their period of "latency" is almost absolutely constant, and their electro-magnets are so wound that no sparking takes place on breaking the circuit.

A moving surface is carried on a carriage, which is propelled by means of a falling weight, which after a certain velocity has been attained is removed: the surface then moves with a velocity which is found to be practically constant for the limits between which a time record is made.

The chronograph is used in conjunction with a steel tube in which the explosions take place. At even distances along the axis of the tube, conducting bridges, eight to ten in number, of Dutch metal insulated from the tube, are placed; each bridge is connected electrically with a recording stylus, so that as each bridge is broken by the explosion, a mark is made on the surface of the chronograph; these markings when duly interpreted provide data for constructing a curve, which indicates the rate at which the velocity of the explosion is changing during its propagation.

The rest of the paper treats of the methods by means of which the errors due to the use of electro-magnets in chronographic work have been dealt with and reduced as far as possible.

Chemical Society, March 7.-Mr. W. Crookes, F.R.S., in the chair. - The following papers were read :-The decomposition of carbon disulphide by shock; a lecture experiment, by Prof. T. E. Thorpe, F.R.S. The author, in studying the action of the fluid alloy of potassium and sodium on carbon disulphide, obtained a yellowish-brown solid substance which exploded with great violence when subjected to pressure or friction. If the explosion occurred in contact with carbon disulphide, that substance was resolved into its elements. A similar decomposition of carbon disulphide into carbon and sulphur can readily be effected by exploding a charge of 0.05 gramme of fulminate within a stout glass tube containing carbon disulphide vapour, and the experiment affords a good illustration for class purpose of the resolution of an endothermic compound into its elements by sudden shock. - The determination of the constitution of the heteronucleal $\alpha \beta$ - and $\beta \beta$-di-derivatives of naphthalene, by Prof. H. E. Armstrong and Mr. W. P. Wynne. A preliminary note on the constitution of the three chloramidonaphthalenesulphonic acids obtained by sulphonating $\alpha$-chloro- $\beta$-amidonaphthalene hydrochloride with weakly fuming sulphuric acid.-The action of chloroform and alcoholic potash on phenylhydrazine, by Dr. S. Ruhemann.

March 28.-Annual General Meeting.-The "following is an abstract of the Annual Report, read by the President, Mr. W. Crookes, F.R.S. :--The pleasant duty again devolves on me to present to you the annual report on the state of the Chemical Society during the twelve months just past. The following statement shows the numerical position of the Society-

Number of Fellows (March 28, 1888) $\quad \ldots \quad \ldots \quad 1534$

Present number of Fellows $\quad \ldots \quad \ldots . \quad \ldots .1614$

Increase 